\title{
Audit: an annual assessment of the work and performance of a surgical firm in a regional teaching hospital
}

\author{
M H GOUGH, M G W KETTLEWELL, C G MARKS, S J K HOLMES, JEAN HOLDERNESS
}

The assessment of the results of treatment has for long been one of the responsibilities of the medical profession. Prospective and retrospective surveys have formed the basis of original and review articles in the medical press, and of lectures and seminars. Postgraduate education has been well served by these traditional methods, which have often led the individual doctor to change and so improve his or her techniques in diagnosis and treatment.

The scene, however, is changing. The cost of medical treatment required to provide a "comprehensive" health service has, for various reasons, outstripped the resources available from the State. As a consequence the allocation and reallocation of finances and personnel have become matters of great importance to administrators, doctors, and politicians. A second change has been in the attitude of the patient, who has become more knowledgeable about health, and if, sometimes, higher expectations of hospital or medical care are unrealised may more often turn to some form of official complaint, or even litigation. Thirdly, the profession itself is changing. The number of graduates is increasing, and their attitude is naturally influenced by the changes in the medical curriculum over the past 20 years: overall there has been a reduction in the emphasis on acute hospital medical and surgical care, which has often been a dominant factor in health care planning in the past.

It would be surprising if demands for the more accurate assessment of clinical performance, related especially to cost, had not been heard. It would be equally surprising if the profession had not responded. Demands there are-for example,

John Radcliffe Hospital, Headington, Oxford

M H GOUGH, Ms, FRCS, consultant surgeon

M GW KETTLEWELL, MCHIR, FRCS, consultant surgeon

C G MARKS, MCHIR, FRCS, senior surgical registrar (Present appointment: consultant surgeon at Royal Surrey County Hospital, Guildford)

$S$ J K HOLMES, FRCS, surgical registrar

JEAN HOLDERNESS, medical secretary why a routine operation such as a hernia repair should entail an inpatient stay of five to seven days in one hospital and one or two in another-and response there has been; medical audit has emerged as a discipline.

A new topic only too often brings a new "language," which sometimes appears to delay acceptance of sound basic ideas. The method of audit, for example, has been classified as process audit-what the physician does for patients-and outcome audit-what happens to the patients. The criteria used are explicit - those determined by group consensus - and implicitthose selected subjectively by individual reviewers. ${ }^{1}$ Nevertheless, even among those particularly interested in the subject of audit-described in the Lancet in 1978 as "Does what you are doing stand up to critical analysis ?"2 - there appears little agreement about the best method. Fernow and $\mathrm{McColl}^{3}$ describe one system based on standard and problem-orientated medical records and a method of scoring clinical performance. This does not altogether overcome the objection of McNerney ${ }^{4}$ that physician-dominated systems of audit tend to exaggerate the value of expensive high quality care and fail to be of sufficient scope to take into account lifestyle and environmental factors, nor does it necessarily affect the outcome. In the United States audit has been national policy for some years through the agency of the Professional Standards Review Organisation. A review of the work of this organisation in 1978 showed disappointingly little evidence of improvements in outcome. ${ }^{5}$ As the cost of setting up the organisation from 1973 to 1976 was reported as $\$ 250$ million and the running cost was $\$ 70$ million in 1976 , this method is inappropriate for NHS needs.

Probably some system of peer review will prove to be the most acceptable method of audit of hospital care. In its most straightforward form this entails frank discussion of treatment and complications within a group of like specialists, and their trainees. Such meetings already occur as part of the postgraduate training programme in many centres, and one report of a year's surgical experience in a teaching hospital was 
given by Irving and Temple in 1976.7 This form of audit, however, may not be comprehensive enough to protect clinical services from the threat of cutbacks for financial reasons or take account of numerous minor clinical problems. As the costs of labour and materials rise the necessity for limiting the availability of free health care becomes only too clear. This unpleasant fact is rarely faced by national and local health authorities, and individual clinicians increasingly find that cancellation of admissions and longer waiting lists for admission and outpatient appointments have forced the responsibility on to them-an unhappy and frustrating state of affairs. It is clearly necessary for the clinician who is faced with demands to reduce clinical services, as has happened in the new Oxford teaching hospital, ${ }^{8}$ to have available not only waiting list details and numbers of patients previously treated, but also details of clinical performance, all of which may be compared with information from other centres. Much data is already collected officially by Hospital Activity Analysis.

It came to our attention that so far as part of our surgical practice in 1977 was concerned the Hospital Activity Analysis figures were inaccurate. The Oxford Regional Health Authority was seeking to estimate the work load of the regional neonatal surgical service at this teaching hospital so that a financial allowance to the area might be considered. Personal records were compared with Hospital Activity Analysis figures, and a discrepancy approaching $20 \%$ was discovered. This fact led us to assess the general surgical work of the firm for the year 1978. We present the result of this retrospective assessment. It has also provided an accurate standard against which the morbidity and mortality statistics, which are recorded prospectively at the weekly meeting of the firm, may be judged.

A statement in the report of the recent Royal Commission that "... although doctors account for only $7 \%$ of NHS workers, they are responsible in the end for initiating most of the expense in the NHS ..." led us to think of some way, other than the statistics of patient stay, bed occupancy, etc, of estimating the economics of clinical work, and perhaps of comparing the output or achievement of similar groups by a non-clinical method. To estimate the value of the firm's work in financial terms we therefore subdivided all operations into "minor," "intermediate," or "major" according to the criteria used by the British United Provident Association (BUPA). We estimated from these operations and from outpatients a hypothetical professional income for the work performed by the firm.

Statistical productivity-whether based on Hospital Activity Analysis or financial criteria-is one thing: the real and important benefits of surgery to the patients and their dependants remain impossible to assess fully, except in the inquiring minds of well-trained doctors and nurses who are concerned with each patient. To quote a report in the Lancet in 1978, "Doctors interested in improving the standard of their work are likely to be those with little fear of medical audit." 2

\section{The firm and its work}

The firm is one of five general surgical firms. At the time of the study in 1978 the teaching hospital was the Radcliffe Infirmary (now it is the John Radcliffe Hospital), and all emergency surgery was performed there. Elective surgery was admitted either to the Infirmary or to the Churchill Hospital. The firm was on emergency take for general surgery each Monday and every fifth weekend. Part of the responsibility carried by the firm was for the regional neonatal service, and emergency duties for this specialty were alternate days and weekends. (Details of the firm and its work continue in miniprint, below.)

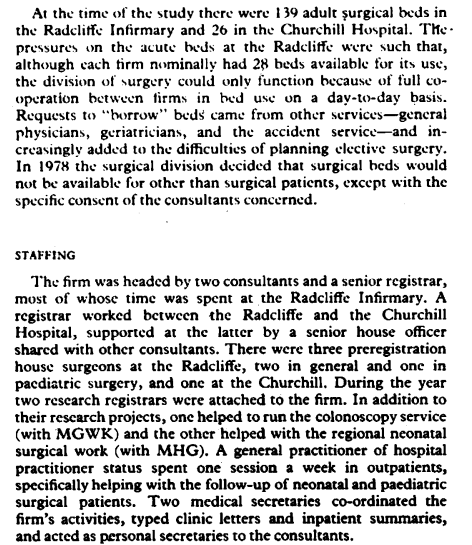

outPatients

The firm held two outpatient sessions a week at the Radcliffe
Infirmary and one a week at a peripheral hospital or health Infirmary and one a week at a peripheral hospital or health
centre. All patients requiring surgery were given an admission
date at the time of their consultation, and this date was rarely date at the time of their cosan swo months ahead.
more than the

TEACHING

Five or six medical students were artached to the firm during the year. They were in either their first or third clinical year.
Four teaching rounds a week were given, supplemented by ward
and theatre teaching. Some years ago it was agreed that the and theatre teaching. Some years ago it was agreed that the
surgical firms should not be too specialised-for example,
rather than have a vascular, a gastroenterological, a urological, rather than have a vascular, a gastroenterological, a urological,
and a paediatric surgical firm, these specialties are spread as far
as possible on each firm. During their two surgical firm atrachas possible on each firm. During their two surgical firm atrach-
ments all undergraduates are therefore likely to be exposed to
the whole range of "ieneral" surgery. Further instruction for the
individual student is provided by a tutorial system organised by individual student is provided by a tutorial system organised by
the medical school. Tutors are drann from the registrars, senio registrars, and lecturers. The firm registrar and
both voluntarily undertook this additional task.

ADMinistration

The business of the firm was discussed at the weekly lunchtime meeting, which was attended by all the medical staff (the included). Dificulties experiing inced in the previous week were
aired and those for the following week anticipated, with paraired and those for the following week anticipated, with par-
ticular regard to planned admissions and the availability of
beds and nurses.
Requests for co-operation or information from the hospital administration were discussed. Holiday and, if necessary, locum
requirements were approved. Holidays were covered within the requirements werc approwed. Holidays werc covered within the
firm, except that a senior medical student might act as locum
during the holiday of a prectegistration house surge a WARD ROUNDS

The senior registrar saw all paticnts at the Radcliffe Infirmary and the registrar those at the Churchill, at least once a day. The whe latter round after the lunchtime meeting. The consultants, senior registrar, and houseman on duty went round each
Saturday morning. Both consultants took teaching rounds each
weck, as did the senior registrar.

THEATRE WORK

Nine theatre sessions a week, seven at the Radcliffe Infirmary and two at the Churchill Hospital, were available and almost a on during the day of admission they were usually accommodat on the next routine list. The firm was fortunate to work mainly in a theatre in the charge of a senior sister who had bee
working with one of us (MHG) for more than ten years. In working with one of us (MHG) for more than ten years. In
addition to the theatre sessions there were two endoscopy
sessions

Methods The number of patients seen and treated by the firm during
1978 was obtained from outpatient records, casualty books, 1978 was obtained from outpatient records, casualty books,
inparient ward admission books, operation records, and dis-
charge summaries. When a patient moved from one department of the hospital to another the accuracy of the data from different
sources was cross-chected. It was decided not to use the case sources was cross-chected. It was decided not to use the case
notes if this could be avoided, and fewer than 100 were required
Two registrars (CGM and SJKH) and one medical secretary Two registrars (CGM and SJKH) and one medical secretary
(JMH) collected the data; this took over 300 "man" hours. For each inpatient the name, hospital number, age, and dates
of admission and discharge, total stay, procedure (with surgeon
when appropriate), postoperative stayy, and diagnosio wererer when appropriate), postoperative stay, and diagnosis were
entered on the proforma for further analysis. All operations were entered on the proforma for further analysis. All operations were
classified as minor, intermediate, and major as described above.
The numbers of patients undergoing different procedures wer obtained from these figures. The median postoperative stay and the time spent in hospital after surgery up to discharge of 90 of patients were calculated for several common operations. meeting of the firm. The complications were arbitrarily divided
into three categories of minor, moderate, and serious, depending into three categories of minor, moderate, and serious, depending
on their effect on the outcome of surgery and whether the on their effect on the outcome of surgery and whether the
patient's stay in hospital was prolonged. Morbidity was also
related to the operative procedure and to the age of the patient, whether adult or child.
Finally, the figures of admissions and operative procedures
obtained by the firm's audit were compared wich those from the obtained by the firm's audity
Hospital Activity Analysis.

oUtPatients

In the three outpatient clinics held each weck a total of
5503 patients were seen. At the Radcliffe Infirmary clinics $30^{\circ}$ " 5503 patients were seen. At the Radclife Infirmary clinics $30 \%$
(1465) of patients attending were new referrals, whereas in the

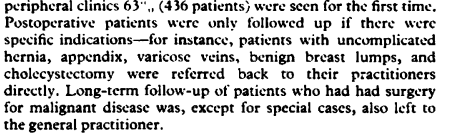
for malignant disease was, except for special cases, also left

INPATIENTS

Our analysis showed that 2607 partients were admitted and treated by the firm, whereas a total of 2138 was recorded by the
Hospitial Activity Analysis. Our figure included 380 patients who
underwent upper or lower gastrointestinal endoscopy. Some of underwent upper or lower gastrointestinal endoscopy. Some of
these patients were referred as inparients already under the care these patients were referred as inparients already under the care
of other firms and therefore may not appear in the Hospital
Activity Analysis figures under our firm. In addition Hospital Activity Analysis figures under our firm. In addition Hospital
Activity Analysis had incomplete information on roughly 7 patients when this analysis was completed in autumn 1979 .
Examination of the casualty records showed that 573 patients were admitited as emerergencises. Of these, 221 were operated on
and 282 were discharged after a period of observation, some and 282 were discharged after a period of observation, some
having later elective surgery. About half of all the patients seen having later elective surgery. About half of all the patients seen
in casualty who did not have an operation suffered from ab-
dominal pain for which no specific cause was found. In addition
to the admissions the casualty records showedt that 100 patients
were alluwed home after examination in casualty.

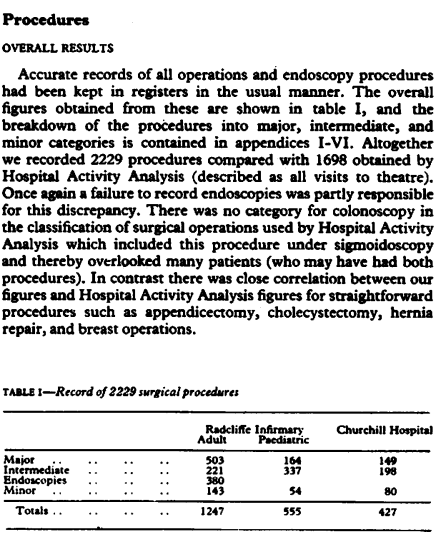
THE SURGEON

For most procedures the name of one surgeon was recorded;
where two surgeons were noted the senior surgeon was considered

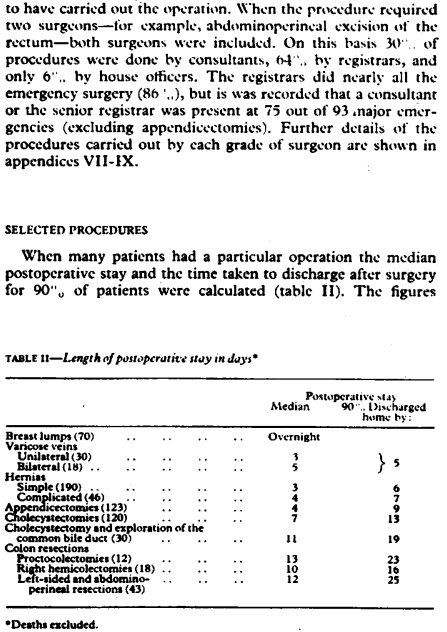

-Desthe excluded.

refer to the numbers of patients on whom all the information refer to the numbers of patients on whom all the information
was avaiable. The median postoperative stay for all conditions
was within an acceptable range, but when the discharge timcs were considered $10^{\circ} \circ$ of the patients undergoing biliary and MORBIDITY

All morbidity that occurred in hospital, however trivial, was recorded, and most episodes were discussed with a view to
improving standards of care. The wekly morbidity shects for
1978 were reviewed and 1978 were reviewed and cach event classified arbitrarily as minor,
moderate, or serious. Wc considered serious morbidity as
potentially life threatening, while moderate morbidity adversely potentially life threatening, while moderate morbidity adversely
affected the parient and probably prolonged the hospital stay.
Minor morbidity, however, was mainly a muisance to both affected the patient and probably prologged the hospitat stay.
Minor morbidity, however, was mainly a nuisance to both
patient and staff. Complications such as intra-abdominal nescess, septicaemia, anastomotic leakage, or hacmorrhage
needing surgery were catcegrised as scrious complications,
while wound, chest, and urinary tract infections, minur hacmorwhile wound, chest, and urinary tract infections, minur hacmor-
rhages treatcd conservativcly, and the removal of a normal
appendix were moderate complications. Examplis of minor appendix were moderate complications. Examplcs of minor
complications were drip-site phlchitis, confusion or depression, ileus, and minor wound hacmatoma. All deaths occurring in hospital were also recorded and
discusscd, even if death was incvitable. 


\section{Results}

Thirty-three patients, of whom five received no operation, died while in hospital. The hospital operative mortality was therefore $1.3^{\circ}$ of all patients. Table III shows the causes of death: the nine patients dying of carcinomatosis were the inevitable result of failure to cure most patients with malignant disease, but five of the nine cases underwent laparotomy in an unsuccessful attempt at palliation.

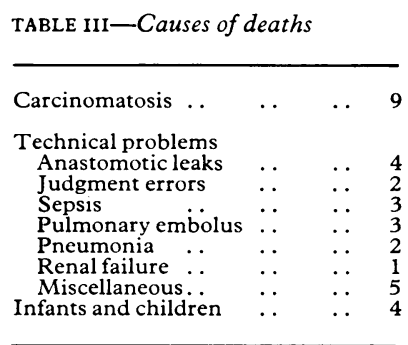

Six patients died as a result of failure of surgical technique or judgment. Four died from anastomotic leakage after gastric or colonic resection. Two died as a result of an error of judgment: one of faecal peritonitis from a longitudinal sigmoid myotomy in an incompletely prepared colon for diverticulitis, which was an incidental finding at laparotomy for repair of rectal prolapse. The other patient, who was in myocardial failure, died after colonoscopy from the effects of diazepam sedation.

Three patients had a fatal pulmonary embolus, an incidence of $0.46 \%$ of all adult major procedures or $0.23 \%$ of all adult procedures. None of these three patients received any form of prophylaxis. Two patients, aged 72 and 63, had undergone elective cholecystectomy and the third, aged 81 , had had a rectal prolapse treated by an Ivalon sponge repair. The policy of the firm at this time was to use subcutaneous heparin prophylaxis in patients at particular risk-for example, those with a previous history of thromboembolism, the obese, and those having treatment with oestrogens, and in advanced malignancy. Using these criteria, none of the three patients who died should have received prophylactic treatment. Three patients died of overwhelming sepsis, two as a complication of acute pancreatitis and one three months after a road traffic accident that had necessitated thoracotomy and splenectomy. Four infants or children died. One had severe spina bifida and was being treated symptomatically. One was cyanosed from birth and died after repair of a diaphragmatic hernia and the third, of low birth weight, died after repair of oesophageal atresia. One death was theoretically avoidable. This child had previously undergone rectosigmoidectomy for Hirschsprung's disease and was readmitted with signs of intestinal obstruction. Initially, the experienced registrar diagnosed that this was recurrent functional obstruction-a not uncommon late postoperative complication-and treated the child conservatively. Laparotomy 24 hours later showed a large gangrenous loop of small bowel, secondary to an adhesion.

If the deaths are considered in relation to the common categories of operation, no patients died after appendicectomy but three $(2.3 \%)$ died after cholecystectomy, one of whom had recurrent cholecystitis and known severe chronic active hepatitis. Five $(7 \cdot 7 \%)$ died after gastric surgery. Two were due to anastomotic leaks after elective surgery for benign gastric ulcers, two followed surgery for perforated gastric ulcer, and one followed perforation of the oesophagus from Celestin intubation for an inoperable carcinoma. One $(0.3 \%)$ died after inguinal hernia repair under local anaesthetic from a combination of pre-existing cardiac, respiratory, and renal failure. This we classified as an avoidable death.

Of the complications of surgery, 66 patients suffered 80 serious complications while there were 197 moderate complications in 164 patients and 59 minor problems in 54 patients (appendices X-XV). Of the adult inpatients, $16.9 \%$ suffered some sort of complication, which was potentially life threatening in $3.9 \%$. Postoperative complications contributed to a delay in discharge from hospital in $13.7 \%$ of patients and may have contributed to about $(230 \times 3=690) 690$ bed days lost.

The infants and children appeared to fare much better than adults for there were only $51(9 \cdot 1 \%)$ complications, of which $18(3 \cdot 2 \%)$ were serious, in 31 children $(5.7 \%)$ (appendix XV). When the serious complications after major surgery are compared, however, $10.1 \%$ of adults and $10.9 \%$ of children were affected: most of these children were neonates undergoing correction of major congenital anomalies.

\section{POSTOPERATIVE SEPSIS}

Intra-abdominal and wound infections were relatively uncommon $(2.4 \%$ of major and $3.1 \%$ of major +intermediate). There were 16 cases of intra-abdominal abscess and 34 of wound infection, of which 12 were in clean wounds, 14 in potentially contaminated, and eight in contaminated wounds. The firm's policy was to give each patient cephradine $1 \mathrm{~g}$ and metronidazole $500 \mathrm{mg}$ intravenously during the operation if the gastrointestinal tract was opened. The antibiotics were continued only if the patient was immunosuppressed-for example, with steroids - or if there was an unusual degree of peritoneal soiling. Infective complications in the common operations are presented in appendix XIII.

Of the 31 episodes of urinary tract infections, 27 were associated with an indwelling catheter. It is not possible to say what proportion of patients with catheters became infected. Of 52 patients with considerable chest infection, 21 were recorded as being heavy smokers (more than 20 cigarettes a day). More than $10 \%$ of patients having major abdominal surgery suffered from chest infections. When all these infective complications are added together an alarming figure results, ranging from $10.3 \%$ after appendicectomy to $40.5 \%$ after colorectal surgery.

Other indices of surgical performance include the incidence of wound dehiscence, anastomotic leaks, retained biliary stones, and errors in diagnosis. Two patients had a wound dehiscence $(0.3 \%$ of all major procedures)-both operations were performed by experienced senior registrars. Of the 11 anastomotic leaks or enterocutaneous fistulas, four led to the patient dying. Three leaks occurred after elective gastric surgery $(4 \cdot 6 \%)$ for benign disease, and two patients died. Two faecal fistulas after laparotomy and drainage of postpancreatitic abscesses and five $(6 \cdot 3 \%)$ faecal leaks after colorectal operations occurred.

Four of 24 patients who underwent exploration of the common bile duct for stones detected by operative cholangiography were found on a later $\mathrm{T}$-tube cholangiogram to have retained stones in the common duct. Eight patients had unnecessary explorations of the common bile duct, two of whom had an unsuccessful peroperative cholangiogram.

Postoperative haemorrhage was an infrequent complication. Three patients needed to be taken back to the operating theatres to arrest haemorrhage, and a further four patients had an appreciable bleed that did not require more than transfusion. There were also eight wound haematomas.

Thirty-five diagnostic errors led to an unnecessary laparotomy in four patients (pseudo-obstruction (1 case), acute pancreatitis (2), and hepatitis (1)). Twenty-three $\left(19^{\circ}{ }_{0}\right)$ appendices when removed were histologically normal.

\section{COSTING OF THE FIRM'S WORK}

The sum of $£ 396240$ (appendix XVI) is an approximate estimate of the work done by the firm when costed according to BUPA scales. We have made no attempt to estimate nursing or other costs initiated by the firm's work, but during 1978 the cost per patient per day at the Radcliffe Infirmary was $£ 58 \cdot 35$, only $10 \%$ of this amount being for medical staff services. The cost per outpatient attendance was $£ 11.75$, $30 \%$ of this amount being for medical staff. Using these figures and taking the median inpatient stay as five days, the cost of providing non-medical services for the 2607 inpatients and 5503 outpatients was therefore about $£ 730000$.

\section{Discussion}

Some aspects of the survey have given cause for satisfaction and some for concern. Among the former is the number of patients (2607 inpatients; 5503 outpatients) we have been able to treat, sometimes under difficult conditions because of shortage of nursing staff or industrial action. We must acknowledge the work of the nursing staff who were almost always able to respond not only to the demands made by our increasingly specialised surgery but also to the apparently endless stream of patients requiring their care. The ward sisters' task seemed often to be made more difficult by changes in the nurse training 
programmes, which left them short of partly trained staff, and by deficiencies in the recruitment of trained staff. Despite these problems, we believe the vast majority of patients who were treated in our wards were well pleased with the care they received. Of the factors that have caused concern, it has been salutary to be reminded of the annual complication rate, even after operations in which the firm could offer particular expertise and remembered as trouble free.

One aim of this survey was to check the Hospital Activity Analysis statistics on which we suspected local resource allocations might partly be based, and we have shown an $18-24 \%$ shortfall. As with many other units, we needed more equipment (in particular fibreoptic endoscopes that were in woefully short supply because of financial stringency) and more secretarial help because of the clerical demands made by the large clinical work load. Secondly, we wished to have accurate figures on which to base our prospectively collected morbidity statistics. Thirdly, with recent papers on medical audit in mind, we had already accepted the need for a more formal method of selfassessment and preferred that this be conducted by ourselves in our own way rather than be, in due course, imposed or perhaps be based only on suspect Hospital Activity Analysis and other figures. Fourthly, in a period of change in the NHS, with the growth of hospital practice being curtailed, we thought that a formal assessment of the work load of a surgical firm in a regional teaching hospital might one day prove to be of historical interest as we could find no other similar published record. The fact that the firm functions as a unit, each consultant knowing the other's patients, and taking over responsibility in the other's absence, and that housemen and registrars are similarly concerned with both consultants' patients, made this a particularly apposite task.

\section{ADMINISTRATION OF THE FIRM}

The weekly meeting, held over an informal lunch, has proved of real value in co-ordinating the firm's activities. It ensures the accuracy of morbidity statistics because relevant events are fresh in the mind. Complications are recorded at the time on proforma. There is no doubt that the apparently high figure of complications- $16.9 \%$ of all adults suffered some sort of complication-is an uncomfortable reality, although it should be emphasised that all variations from the norm were recorded. It is certainly higher than we expected. The $3.9 \%$ of adult patients who had a life-threatening complication might have been accurately recalled in a retrospective survey, although even these serious events may slip from memory; the $13 \%$ who had minor or moderate complications would certainly have been underestimated.

A system of giving a patient the date for admission when seen in outpatients is the policy of the firm and has been practised by one of us (MHG) since 1967. It is a great help to the patient for many obvious reasons. It allows planning of operating lists so that they are balanced in length and with regard to type of operationoften important when teaching registrars or medical studentsand also takes into account consultant and registrar holidays and absences, which are noted in the operation diary as soon as they are known. The major drawback is when admissions have to be cancelled-usually because of a heavy emergency load, incursions into the firm's beds by other firms, or nursing shortages. If patients are cancelled after an admission date has been arranged they sometimes seem more aggrieved than when they have been given only short notice of admission. It is also difficult with this booking system to fit in the cancelled patient within the next few weeks. In sum, however, the advantages far outweigh the disadvantages. A "statistical" disadvantage should be mentioned, as it may lead to misunderstandings on the part of those administrators who rely on "bed occupancy" statistics based on returns taken at midnight. With planned elective admissions beds are empty overnight but occupied the next morning, with resulting, apparent, under-usage. This form of assessment of bed occupancy also took no account of day-case patients who were admitted to the normal ward because of lack of special facilities.

\section{OUTPATIENTS}

The members of the firm saw some $30 \%$ of all the new patients seen at surgical outpatients at the Radcliffe Infirmary. This high proportion is partly explained by the number of children seen with relatively minor complaints-for instance, hydrocele, hernia, and undescended testes. Our practice regarding followup of postoperative patients has already been mentionedwithout this policy numbers attending the clinic would have been excessive, and the standard of care available for those who did need hospital follow-up would have deteriorated. A particular group requiring follow-up were those children who had undergone surgery for major congenital defects. Although the regional neonatal service is conducted from Oxford, there is no paediatric surgical registrar or senior registrar. Many of the regional referrals are routinely followed up at their local hospital with twice or thrice yearly visits to Oxford. A hospital practitioner grade general practitioner has helped with this followup and provided the necessary continuity of care for growing child and parents alike. There has been much discussion, and even concern, over the relevance of this grade of practitioner in the surgical specialties. As a part-time member of the firm, our general practitioner has given invaluable help.

Among patients arriving at the casualty and emergency department there was a small additional outpatient work load that was not represented in official statistics unless the patient later returned to the outpatient department. During the audit 100 patients presented as emergencies and were examined and sent home to the care of their own practitioner.

\section{MORTALITY}

Care provided by well-staffed intensive care units for both adults and children was important in reducing mortality and morbidity. The method of recording the incidence of both these factors has already been mentioned. Further comment is required on the figures themselves. The mortality figures are accurate in terms of death for causes related to surgery but underestimate the overall number, the reason being that, if possible, patients known to be in their terminal illness were referred home to the care of their relatives and general practitioner or, failing that, to Sir Michael Sobell House, the excellent continuing care unit at the Churchill Hospital (Dr R Twycross). For those remaining under our care every attempt was made to ensure that death occurred in a comfortable and dignified way, although in a busy, and at times over-busy, old general surgical ward conditions were less than ideal for patient and relatives.

Six patients died as a result of an error of judgment or surgical technique. The operations of high gastric resection and low colonic resection have a notorious reputation for anastomotic leakage, but dispassionate analysis of these cases must attribute at least part of this incidence to the relative inexperience of the surgeon and failure of supervision. A new registrar joins the firm each six months (the rotation being six six-monthly posts) and the senior registrar rotates annually. The firm's policy is that trainees first help at the various operations, then are helped by one of the consultants, and when it is considered appropriate they take full responsibility for the operation. Even then further supervision is available because one of the consultants (MHG) and the trainee operate in adjacent theatres on one day of the week, and, with the booking system for admissions, appropriate cover may be arranged for the technically more difficult operation.

One patient died because of inadequate assessment before colonoscopy. This woman, who was one of $15 \%$ of colonoscopy 
patients referred from outside the area, was an ill-advised choice for referral. The subsequent assessment was by an inexperienced house surgeon, and the endoscopist did not appreciate the gravity of her condition before colonoscopy.

\section{MORBIDITY}

The wound infection rate was agreeably low. Nevertheless, postoperative infections of the chest and urinary tract in those patients who had been catheterised was too high. Preoperative physiotherapy was given, when appropriate, to all patients undergoing elective surgery. Cigarette smoking remained the single most obvious predisposing cause of morbidity, and on occasions patients were refused operation until their smoking habits and chest signs improved. Throughout the year of audit there was a considerable shortage of physiotherapists available on the surgical wards, and despite efforts from medical and nursing staff to make good the deficiency this factor contributed to postoperative morbidity. The incidence of urinary tract infection in catheterised patients is based on bacteriological examination of the urine just before catheter removal. Not all these patients had symptoms, but all were treated with the appropriate antibacterial agent.

An unexpectedly, and unhappily, high incidence (17\%) of retained common bile duct stones after exploration was shown. In two patients a calculus was knowingly left in an intrahepatic duct, apparently impacted. In the other two patients the retained stones were shown by $\mathrm{T}$-tube cholangiography one week postoperatively. Peroperative cholangiography was routine, but this incidence led us to press for the purchase of a choledochoscope. If there was still doubt we performed a T-tube cholangiogram at the end of the common duct exploration. The T-tube inserted was of appropriate size and so positioned that extraction of a retained stone by the Burhenne method was later possible.

\section{COSTING}

Whether costing of surgical work by the method we have described will prove of value in assessing resource allocation rather than be just a matter of passing interest, is difficult to foretell. The more efficient a surgeon or group of surgeons, the more readily should resources be available, but this is not always the case, and sometimes it seems that there is little recognition either of efficiency or inefficiency when staff or financial allocations are planned.

\section{Conclusions}

We believe that it would help to tabulate our conclusions, although we realise that there are disadvantages in appearing to be over-definite in our inferences from this study.

(1) A formal, prospective audit by the firm or division should be a routine part of surgical practice. Accurate retrospective audit is too time consuming and may also result in delay in improving surgical or administrative practice.

(2) The present clinical secretarial establishment is not adequate for this task and additional administrative support should be available at the clinical level. Personnel should perhaps be seconded on a part-time basis from the departments presently responsible for Hospital Activity Analysis. This interchange of personnel would result in better mutual understanding of the clinical and statistical problems and would provide a more accurate analysis and perhaps a more efficient allocation of resources. This audit of one of five firms at this teaching hospital suggests that surgery, as a specialty, may be underfunded.

(3) The use of computers at the clinical level-based on units of either personal, firm, or divisional work-suggests itself as the most efficient way of conducting a prospective audit. Pilot

studies, with specialist advice from Hospital Activity Analysis departments, should be started to explore this idea, which would also minimise the collection of identical data by separate bodies.

(4) The highly technical nature of audit information demands that it should be assessed by doctors in the same, or like, specialties. The considerable influence, however, of social and environmental factors on audit results-for example, the availability of domiciliary and hospital geriatric facilities for postoperative patients, nurse staffing shortages, "industrial action" by hospital staff, etc-requires expert advice not of a surgical nature, and this advice might reasonably be provided by the regional or area (district) medical officer or someone nominated by them.

(5) The aims of audit-to improve patient care, to be part of postgraduate surgical training, to help ensure the efficient use of increasingly scarce resources-must not be undermined by improper use of audit information. Confidentiality within the audit team, or hospital group concerned, must be secure, for

\section{MINIPRINT APPENDICES I-XVI}
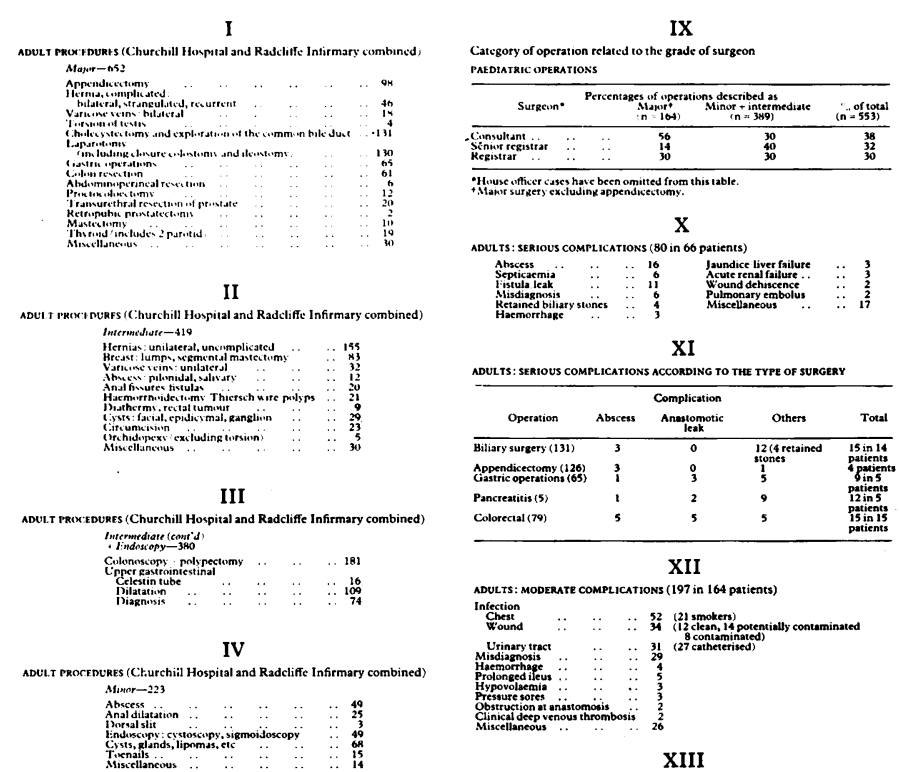
there are dangers that frank assessment could be used as a source for overdramatic coverage in the lay media. The inevitable result of this would be that frankness, and audit itself, would disappear, and the potential for improving care that might accrue for patients in the future would be lost. By approaching audit in a "scientific" way as part of the postgraduate education of trainee and established surgeon alike, the understandable reluctance of appearing to judge or criticise one's colleagues will almost certainly be overcome, although this considerable change in surgical practice will probably take several years to effect.

(6) We believe that the work load shown by this audit is unreasonably large for the staff concerned. The complication rates might have been improved by a lesser load and by closer consultant supervision of diagnosis and operative technique, both in emergency and elective surgery than time currently allows. Although many well-trained surgeons await consultant appointments, national resources unfortunately allow only a few new posts to be established. The pattern of surgical work between trainee and consultant, which has become established since the inception of the NHS, and the relationship of the NHS to private consultant surgical practice probably temper the demands to DHSS from the surgical profession for new consultant appointments. A high "throughput" is demanded by the number of patients seeking care and is popular among trainees because it provides much experience in a short time. It does not necessarily produce the best environment for a balanced scientific and practical surgical training, and it may not produce the best results for the individual patient.

ADDENDUM.-Since submission of this article the surgical beds at the John Radcliffe Hospital have been reduced by 20 for financial reasons.

\section{References}

1 Brook RH, Appel FA. Quality-of-care assessment: choosing a method for peer review. N Englf Med 1973;288:1323-9.

2 Anonymous. Medical audit. Lancet $1978 ; \mathrm{i}: 1166$.

${ }^{3}$ Fernow LC, McColl I. The state of British medicine: medical audit. f R Soc Med 1978;71:787-90.

${ }^{4}$ McNerney WJ. The quandary of quality assessment. $N$ Engl f Med 1976; 295:1505-11.

5 Anonymous. $\mathrm{Br} \mathrm{Med} \mathcal{F}$ 1978;ii:156.

"Hall H. Medical audit: say "no" to audit. World Medicine 1979;14:21-2.

7 Irving $M N$, Temple J. Surgical audit: one year's experience in a teaching hospital. Br Med f 1976;ii:746-7.

8 Oxford Mail 1979 Oct 24.

\title{
Occasional Review
}

\section{Trials and tribulations: thoughts on the organisation of multicentre clinical studies}

\author{
CANCER RESEARCH CAMPAIGN WORKING PARTY*
}

\section{Summary and conclusions}

The Cancer Research Campaign Trial concerning the management of early breast cancer recruited well over 2000 patients, thus making it the largest prospective study of the management of cancer on record. The management of such large numbers created specific problems of organisation. It is recommended that the protocol design and proforma should be kept simple. A centralised secretariat should be set up, and statisticians and computer programmers must be concerned at every step, including design and analysis. Large clinical trials are very expensive and should not be embarked on without adequate long-term finance.

* Working party members: Professor Michael Baum (chairman); Dr John I Haybittle (vice-chairman and project statistician); Mr David A Berstock; Dr Diana M Brinkley; Dr Christopher W Elston; Professor G Austin Gresham; Mrs Joan Houghton (trial co-ordinator); Miss Gillian Kearney (computer programmer); Mr D Paul Leiberman; Mr John MacIntyre; Professor Joseph S Mitchell (past chairman); Professor J Greig Murray (past chairman); Dr Gita S Rao; Mr William Ross; Mr Jack Thirlwal (computer scientist); and Dr Terence Wheeler. (Co-ordinated by: Departmen of Surgery, King's College Hospital Medical School, London SE5 8RX.)

\section{Introduction}

The Cancer Research Campaign (CRC) Trial on early breast cancer ${ }^{1}$ is now in its 10th year of follow-up, and a full report on its analysis is in press. ${ }^{2}$ We believe that, since this is one of the largest clinical trials undertaken, the problems encountered and overcome during this period should be reviewed, since many are common to all clinical trials demanding long-term follow-up of large numbers of patients. ${ }^{3}$

The trial was designed specifically to minimise type II error ${ }^{4}-$ that is, a conclusion that there is no difference between the treatment arms when in fact a real difference does exist-and therefore it was planned to recruit over 1000 patients in each of the two treatment arms. This necessitated recruitment from as many centres as possible, and the protocol was designed with this in mind.

\section{Protocol design}

The study was successful in that recruitment exceeded the required 2000 patients. This can largely be attributed to the fact that the question asked by the protocol was one that was uppermost in clinicians' minds 10 years ago-namely, "What is the relevance of treating regional lymph nodes at the time of mastectomy ?" Many clinicians working in district hospitals were enthusiastic about the possibility of collaborating in a large international study, and this enthusiasm was maintained because of the simple design of the 